\title{
Tecnología de membrana con enrejados tipo zeolita
}

\author{
J. Vega Moreno', E. Reguera ${ }^{1}$, J.A. I. Díaz Góngora ${ }^{1}$, \\ A. A. Lemus Santana ${ }^{1}$
}

\begin{abstract}
Resumen: Hoy en día, las plantas existentes de energía a base de carbón presentes en el mundo emiten cerca de 2 billones de toneladas de $\mathrm{CO}_{2}$ al año. Debido a su ingeniería fundamental y las ventajas económicas que compiten en tecnologías de separación, las operaciones de membrana están siendo exploradas para la separación de $\mathrm{CO}_{2}$ y otros gases de combustión de las emisiones de centrales eléctricas basados en combustibles fósiles. [1] Los enrejados imidazolato tipo zeolita (ZIFs) son nuevos materiales microporosos con diversidad de estructuras y tamaños de poros, así como grandes áreas superficiales. Las membranas de estos materiales poseen un gran potencial para la separación de mezclas de gases de interés energético y ambiental. [2-4]

En este trabajo se presenta la síntesis y caracterización de dos membranas potencialmente útiles para separación de $\mathrm{CO}_{2}$, a partir del compuesto conocido como ZIF-8 y una modificación del mismo usando una mezcla de metales ( $\mathrm{Zn}$ y Co). Con esta modificación se espera modular la entalpía de interacción y con ello a su vez, la selectividad de la membrana como resultado de la participación de dos tipos de metales en el enrejado molecular.
\end{abstract}

ABSTRACT: Today, existing power plants based on coal present in the world emit about 2 billion tons of $\mathrm{CO}_{2}$ per year. Because of its fundamental engineering and economic advantages in competing separation technologies, membrane operations are being explored for the separation of $\mathrm{CO}_{2}$ and other exhaust emissions of power plants based on fossil fuels. [1] The zeolite imidazolate framework (ZIFs) are novel microporous materials with different structures and pore sizes and large surface areas. Membranes of these materials have great potential for the separation of gas mixtures energy and environmental interest. [2-4]

This paper reports the synthesis and characterization of two potentially useful membrane separation of $\mathrm{CO}_{2}$ from the compound known as $\mathrm{ZIF}-8$ and an amendment thereto using a mixture of metals ( $\mathrm{Zn}$ and $\mathrm{Co})$. This modification is expected to modulate the interaction enthalpy and thereby turn the selectivity of the membrane as a result of the involvement of two types of metals in the molecular lattice.

\section{INTRODUCCIÓN}

En los últimos años se han reconocido potenciales ventajas económicas, ambientales y de implementación en la separación de mezcla de gases (SMG) utilizando la tecnología de membrana debido a que es un proceso de régimen semiestacionario; el cual no requiere aditivos ni tratamientos secundarios, en comparación con otras tecnologías de SMG (por ejemplo absorción con aminas y destilación criogénica).

Entre los diversos mecanismos de separación descritos para las membranas, se encuentra el tamizado molecular. Dicho mecanismo se halla favorecido en los materiales microporosos (hasta $2 \mathrm{~nm}$ ).

\footnotetext{
1 Centro de Investigació en Ciencia Aplicada y Tecnología Avanzada, Unidad Legaria, IPN.
} 
La alta regularidad en forma y tamaño que exhiben los nanoporos en estructuras cristalinas de enrejados tipo imidazol (Zeolite Imidazol Frameworks, ZIF's por sus siglas en inglés), así como la estabilidad química y térmica que presentan estos materiales, los hace muy atractivos para ser implementados en tecnología de membrana, y potencialmente útiles para la separación de mezclas de gases y vapores.

En especial el ZIF-8 es prototipo de este tipo de enrejados en donde cuatro anillos del ligante 2-metilimidazol (MeIm) se coordinan a un átomo tetraédrico de zinc formando un enrejado poroso con estructura tipo sodalita (SOD) en la cual hay grandes cavidades $(11.6 \AA$ ) y pequeñas aperturas de poro (3.4 ̊̊). [4]

La fase activa de la membrana esta conformada por ZIF's y es donde recae la eficiencia en la SMG. La fase inactiva es un soporte de $\mathrm{g}-\mathrm{Al}_{2} \mathrm{O}_{3}$.

\section{Metodología eXPerimental}

Los reactivos utilizados para la obtención del ZIFs son los siguientes: metanol $\left(\mathrm{CH}_{3} \mathrm{OH}\right.$ $>99 \%$ Fermont), 2-metilimidazol $\left(\mathrm{C}_{4} \mathrm{H}_{6} \mathrm{~N}_{2}>99 \%\right.$ Aldrich), formiato de sodio (HCOO$\mathrm{Na}>99 \%$ Reasol), cloruro de cinc $\left(\mathrm{ZnCl}_{2}>99 \%\right.$ Fermont$)$ y cloruro de cobalto $\left(\mathrm{CoCl}_{2}\right.$ $>99 \%$ Baker).

Síntesis de los soportes de g- $\mathrm{Al}_{2} \mathrm{O}_{3}$.- Se hicieron pastillas de g- $\mathrm{Al}_{2} \mathrm{O}_{3}$ (fase inactiva) y se colocaron en una estufa a $200^{\circ} \mathrm{C}$ durante $2 \mathrm{~h}$. Sobre la superficie de ellas se goteó una disolución metanólica de 2-metilimidazol (MeIm) a $0.4 \mathrm{M}$, para promover centros de anclaje Al-N. Con esta activación, la síntesis de la fase activa de la membrana se lleva a cabo vía solvotermal.

Síntesis de la películas de ZIF sobre los soportes de g- $\mathrm{Al}_{2} \mathrm{O}_{3}$.- Se prepararon disoluciones precursoras de ZIF-8: $\mathrm{ZnCl}_{2}$ y MeIm en concentraciones equimolares. Para el caso del ZIF-8 mix (Zn y Co), se preparó adicionalmente una disolución de $\mathrm{CoCl}_{2}$. Se vertieron las disoluciones en autoclaves de teflón, sobre los soportes de ã- $\mathrm{Al}_{2} \mathrm{O}_{3^{2}}$. El crecimiento de los cristales de ZIF's se dio luego de $4 \mathrm{~h}$ a $120^{\circ} \mathrm{C}$. Una vez concluido el tiempo de síntesis, la membrana asimétrica se saca de la autoclave, se lava con metanol y se deja secar a temperatura de cuarto.

\section{RESULTADOS Y ANÁLISIS}

Se obtuvieron dos membranas asimétricas que como soporte tienen una fase mesoporosa de $\mathrm{g}-\mathrm{Al}_{2} \mathrm{O}_{3}$ y como fase activa tienen un compuesto ZIF.

Para el caso de la primera membrana, sólo se usó el metal Zinc y su abreviación es: ZIF-8 (Zn) y en el caso de la segunda membrana, se usó una combinación de dos metales: Zn - Co y se abrevió ZIF-mix (Zn-Co).

La caracterización preliminar que se hizo por DRX indicando que las fases de ZIF que cristalizaron en la superficie de la $\mathrm{g}-\mathrm{Al}_{2} \mathrm{O}_{3}$ y el patrón corresponde a la estructura del compuesto reportado de ZIF-8 microcristalino, figura 1. [4]

En la figura 2 se observa una micrografía de barrido (SEM), de la película de ZIFmix (Zn-Co) en una fase continua, y homogénea.

La tabla 1 resume el análisis de espectroscopia de energía dispersiva (EDS) en el que se muestra la relación de metales que se ha obtenido en la fase activa de la membrana. 
FIGURA 1. Patrones de difracción de ZIF-8 (Zn) y ZIF-mix (Zn-Co)

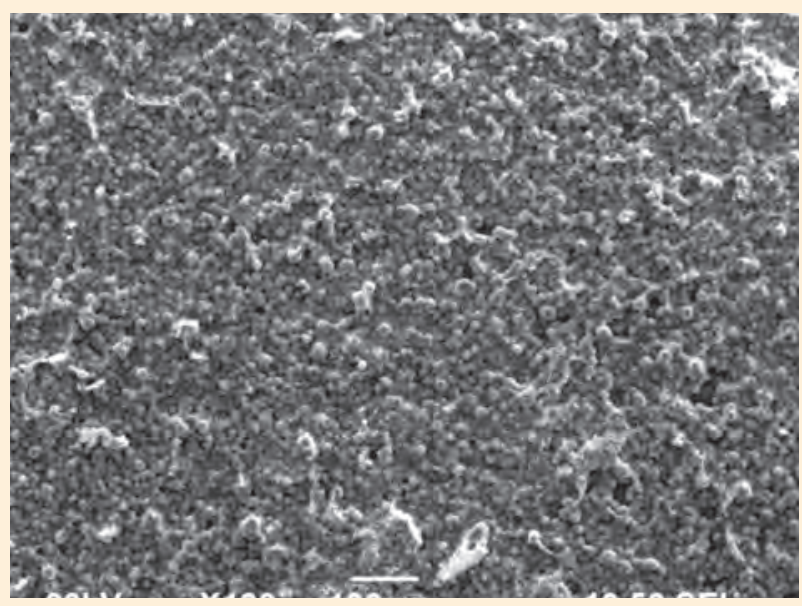

FIGURA 2. Superficie de la fase activa de ZIF-mix (Zn-Co)

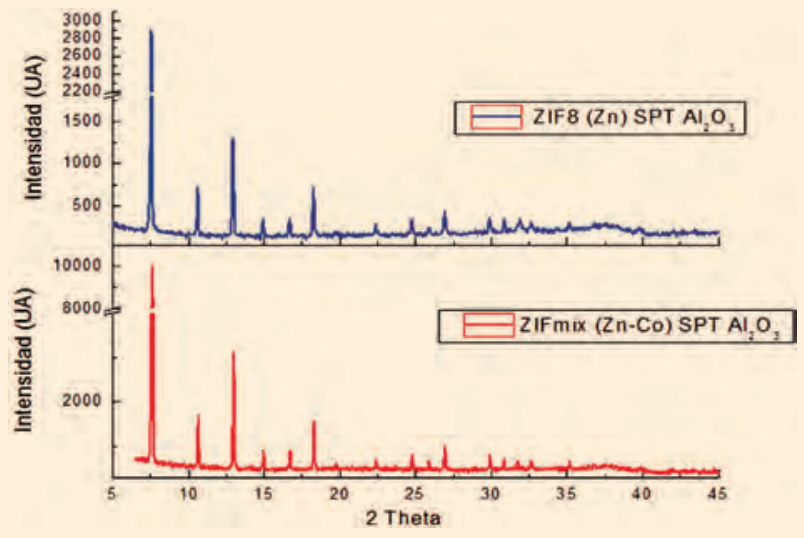

TABLA1. Relación de metales Zn:Co es cercana a 1:1.

\begin{tabular}{|c|c|c|}
\hline Element & Weight $\%$ & Atomic\% \\
\hline $\mathbf{C}$ & 41.74 & 54.24 \\
\hline $\mathbf{N}$ & 28.85 & 32.15 \\
\hline $\mathbf{O}$ & 6.77 & 6.60 \\
\hline $\mathbf{A l}$ & 4.00 & 2.31 \\
\hline Co & 9.47 & 2.51 \\
\hline Zn & 9.17 & 2.19 \\
\hline Totals & 100.00 & \\
\hline
\end{tabular}




\section{Conclusiones}

Se logró el crecimiento de una fase cristalina de enrejados tipo zeolita sobre soportes de alúmina mesoporosa.

Se promueve un crecimiento uniforme del ZIF-8 (Zn) y ZIF-mixto (Zn-Co) en forma de membrana sobre el soporte de $\gamma$-alúmina.

Se logró la incorporación de dos metales en la misma estructura cristalina del ZIF8 y con ello se obtuvo ZIF mix (Zn-Co) como fase activa de la membrana asimétrica potencialmente útil en la separación de mezclas de gases.

Se concluye que el ZIF-mix es isoestructural al ZIF-8 de acuerdo a los resultados obtenidos de difracción de rayos $\mathrm{x}$.

El estudio de la selectividad de ambas membranas asimétricas frente a distintos gases permitirá evaluar la modificación de superficie que la mezcla de metales induce en el enrejado poroso.

\section{AGRADECIMIENTOS}

A los proyectos SIP-20120145, CONACYT 2012-1601 y SEP-CONACYT -CB 2010-2012 166387. JVM agradece a CONACYT por la beca otorgada para sus estudios de maestría. Los autores agradecen a Alfonso Martínez y a Erwin Gómez por el apoyo en los análisis de Microscopía y DRX respectivamente.

\section{Referencias}

[1] Kyo Park, Zheng Ni, Yong Choi, Omar Yaghi. (2006). Exceptional chemical and thermal stability of ZIF PNAS 10310186.

[2] Helge Bux, Armin Feldhoff, Janosch Cravillon, Yan-Shuo Li. (2011). Oriented ZIF-8 Membrane with sharp H2/C3H8 molecular sieve separation Materials 23: 2262-2269

[3] Michael McCarthy, Victor V. Guerrero, Gregori V. Barnett, y Hae-Kwon Jeong. (2010). Synthesis of ZIF films and membranes with controlled microstructures. Langmuir, 26: 14636-14641

[4] Miral Shah, Hyuk Taek Kwon, Vu Tran, Sonny Sachdeva, Hae-Kwon Jeong. (2013). "One step in situ synthesis of supported zeolitic imidazolate framework ZIF-8 membranes: Role of sodium formate", Science 165: 63-69 